OPEN ACCESS

Edited by:

Jie Li,

Harbin Institute of Technology, China

Reviewed by:

Yinan Jiang,

University of Pittsburgh, United States

Vikram Dalal,

Washington University in St. Louis,

United States

*Correspondence: Omayma O Abdelaleem dr.omayma@yahoo.com

Specialty section:

This article was submitted to Molecular Diagnostics and

Therapeutics,

a section of the journal

Frontiers in Molecular Biosciences

Received: 14 August 2021 Accepted: 24 December 2021

Published: 14 February 2022

Citation:

Abdelaleem OO, Shaker OG,

Mohamed MM, Ahmed TI,

Elkhateeb AF, Abdelghaffar NK, Ahmed NA, Khalefa AA, Hemeda NF and Mahmoud RH (2022) Differential

Expression of Serum TUG1, LINC00657, miR-9, and miR-106a in Diabetic Patients With and Without Ischemic Stroke.

Front. Mol. Biosci. 8:758742. doi: 10.3389/fmolb.2021.758742

\section{Differential Expression of Serum TUG1, LINC00657, miR-9, and miR-106a in Diabetic Patients With and Without Ischemic Stroke}

\author{
Omayma O Abdelaleem ${ }^{1 *}$, Olfat G. Shaker ${ }^{2}$, Mohamed M. Mohamed ${ }^{3}$, Tarek I. Ahmed ${ }^{4}$, \\ Ahmed F. Elkhateeb ${ }^{5}$, Noha K. Abdelghaffar ${ }^{6}$, Naglaa A. Ahmed ${ }^{7}$, Abeer A. Khalefa ${ }^{7}$, \\ Nada F. Hemeda ${ }^{8}$ and Rania H. Mahmoud ${ }^{1}$ \\ ${ }^{1}$ Department of Medical Biochemistry and Molecular Biology, Faculty of Medicine, Fayoum University, Fayoum, Egypt, \\ ${ }^{2}$ Department of Medical Biochemistry and Molecular Biology, Faculty of Medicine, Cairo University, Giza, Egypt, ${ }^{3}$ Department of \\ Internal Medicine, Faculty of Medicine, Cairo University, Giza, Egypt, ${ }^{4}$ Department of Internal Medicine, Faculty of Medicine, \\ Fayoum University, Fayoum, Egypt, ${ }^{5}$ Department of Critical Care, Faculty of Medicine, Fayoum University, Fayoum, Egypt, \\ ${ }^{6}$ Department of Clinical Pathology, Faculty of Medicine, Fayoum University, Fayoum, Egypt, ${ }^{7}$ Department of Physiology, Faculty of \\ Medicine, Zagazig University, Zagazig, Egypt, ${ }^{8}$ Department of Genetics, Faculty of Agriculture, Fayoum University, Fayoum, Egypt
}

Background: Ischemic stroke is one of the serious complications of diabetes. Noncoding RNAs are established as promising biomarkers for diabetes and its complications. The present research investigated the expression profiles of serum TUG1, LINC00657, miR-9, and miR-106a in diabetic patients with and without stroke.

Methods: A total of 75 diabetic patients without stroke, 77 patients with stroke, and 71 healthy controls were recruited in the current study. The serum expression levels of TUG1, LINC00657, miR-9, and miR-106a were assessed using quantitative real-time polymerase chain reaction assays.

Results: We observed significant high expression levels of LINC00657 and miR-9 in the serum of diabetic patients without stroke compared to control participants. At the same time, we found marked increases of serum TUG1, LINC00657, and miR-9 and a marked decrease of serum miR-106a in diabetic patients who had stroke relative to those without stroke. Also, we revealed positive correlations between each of TUG1, LINC00657, and miR-9 and the National Institutes of Health Stroke Scale (NIHSS). However, there was a negative correlation between miR-106a and NIHSS. Finally, we demonstrated a negative correlation between LINC00657 and miR-106a in diabetic patients with stroke.

Conclusion: Serum non-coding RNAs, TUG1, LINC00657, miR-9, and miR-106a displayed potential as novel molecular biomarkers for diabetes complicated with stroke, suggesting that they might be new therapeutic targets for the treatment of diabetic patients with stroke.

Keywords: TUG1, LINC00657, miR-9, miR-106a, stroke 


\section{INTRODUCTION}

Diabetes mellitus (DM) is a complex, multisystem disease and is one of the risk factors of stroke. Oxidative stress occurs due to an elevated blood glucose level, which is associated with increased glycated end products, resulting in endothelial dysfunction, cerebrovascular atherosclerosis, and thrombosis, which are the main causes of ischemic stroke in diabetic patients that is associated with high mortality and poor prognosis (Nakagami et al., 2005; Ferreiro et al., 2010).

It is important to understand the molecular mechanisms of cerebral stoke associated with DM to facilitate the development of new effective potential biomarkers and therapeutic targets for diabetic patients with stroke.

Non-coding RNAs, including microRNAs (miRNAs) and long non-coding RNAs (lncRNAs), have been proven to have necessary roles in regulating gene expression (Kitagawa et al., 2013; Iyengar et al., 2014; Khoshnam et al., 2017). LncRNAs are an important group of non-coding RNAs that are of long transcripts ( $>200 \mathrm{bp}$ ). Numerous studies have elucidated the significant roles of lncRNAs in various diseases, including ischemic stroke (Mercer and Mattick, 2013; Bao et al., 2018a).

Taurine-upregulated gene 1 (TUG1), a lncRNA, has been shown to be related to the pathogenesis of many diseases. Recently, TUG1 has gained significant attention in ischemic injuries (Long et al., 2016; Chen et al., 2017; Wang et al., 2017), although little has been identified regarding its role in DM complicated with stroke.

LINC00657 is a lncRNA that is highly conserved and profusely expressed in endothelial cells (Michalik et al., 2014). Accumulating evidence has demonstrated that LINC00657 might play an oncogenic role, and it is upregulated in many cancers (Liu H. et al., 2016; Liu S. et al., 2016). However, its role in DM or diabetes-related complications has not been investigated yet.

MicroRNAs (miRNAs) are tiny non-coding RNAs (20-25 nucleotides long). Recently, promising research studies have explained the importance of miRNAs in the pathogenesis of diabetes and its cardiovascular complications (Meng et al., 2012; Koutsis et al., 2013). However, the expressions of miR-9 and miR$106 a$ in diabetic patients with ischemic stroke have not been examined.

Bioinformatics has reported that TUG1 has complementary sequences of miR-9. Additionally, LINC00657 contains binding sites for miR-106a (Li et al., 2014). However, a study of their relationship in DM complicated with cerebral stroke remains to be conducted.

In this study, we aimed to assess the serum expression levels of TUG1, LINC00657, miR-9, and miR-106a in diabetic patients who had stroke and those without cerebral stroke and to explore any association between these non-coding RNAs and clinicolaboratory data.

\section{Subjects and Methods Study Population}

A total of 152 diabetic patients (with type 2 diabetes) were recruited among those admitted to the outpatient and inpatient clinics of the Internal Medicine and Intensive Care
Unit, Fayoum University Hospital, Fayoum, in the period from November 2019 to December 2020. Diabetic patients were selected based on the American Diabetes Association 2015 diagnostic criteria (Pinsker et al., 2015). Diabetic patients were divided into two groups: diabetic patients with stroke (30females and 47 males, with a mean $=57.08 \pm 16.31$ years) and diabetic patients without stroke (26 females and 49 males, mean age $=53.19 \pm 17.78$ years) (Figure 1)

Ischemic stroke diagnosis was assessed according to clinical symptoms and physical examinations, and this diagnosis was confirmed by computed tomography (CT) or magnetic resonance imaging (MRI). The National Institutes of Health Stroke Scale (NIHSS) was used by experienced neurologists to evaluate the neurological deficits.

All patients with brain tumors, intracerebral hemorrhage, recurrent stroke, history of hypertension, recent head injuries, immune system disorders, liver or renal diseases, blood diseases, acute infectious diseases, or a family history of stroke were excluded from the study. Furthermore, 71 healthy individuals (age and sex matched to the patients) who did not have systemic or neurologic diseases were considered as the control group in this study.

Written informed consent was signed by all enrolled participants after a detailed explanation of the study. The study protocol was performed in agreement with the Declaration of Helsinki. The Ethics Committee of the Faculty of Medicine, Fayoum University, approved this research protocol.

\section{Serum Collection}

From each participant, $5 \mathrm{ml}$ of venous blood was collected into plain tubes using a Vacutainer system following a 12-h fast. Serum separator tubes were used to collect the samples that were left for $15 \mathrm{~min}$ to clot. Centrifugation at $4,000 \times g$ for $10 \mathrm{~min}$ was performed, separating the serum that was stored at $-80^{\circ} \mathrm{C}$ until the time of use. An extra blood sample was taken $2 \mathrm{~h}$ after a meal ( $2 \mathrm{~h}$ post-prandial, $2 \mathrm{hPP})$ into tubes containing fluoride.

Fasting blood glucose (FBG), 2hPP blood glucose, cholesterol, triglycerides, HbA1C, creatinine, and low-density lipoprotein (LDL) were assessed using standard methods on cobas c311 (Roche, Mannheim, Germany) in accordance with the instructions in the kit. Serum samples were used for the quantification of TUG1, LINC00657, miR-9, and miR-106a using real-time PCR.

\section{LncRNA and miRNA Extraction and Reverse Transcription}

According to the manufacturer's protocol, total RNA (including lncRNAs and miRNAs) was extracted from serum samples using the miRNeasy extraction kit (Qiagen, Hilden, Germany) after adding the QIAzol lysis reagent. Quantitation and the purity of the RNA samples were assessed using the NanoDrop ${ }^{\circledR}$ (ND)-1000 spectrophotometer (NanoDrop Technologies, Inc., Wilmington, DE, USA).

Complementary DNA (cDNA) was generated from the extracted RNA in a total volume of $20 \mu \mathrm{l} /$ reaction using the 


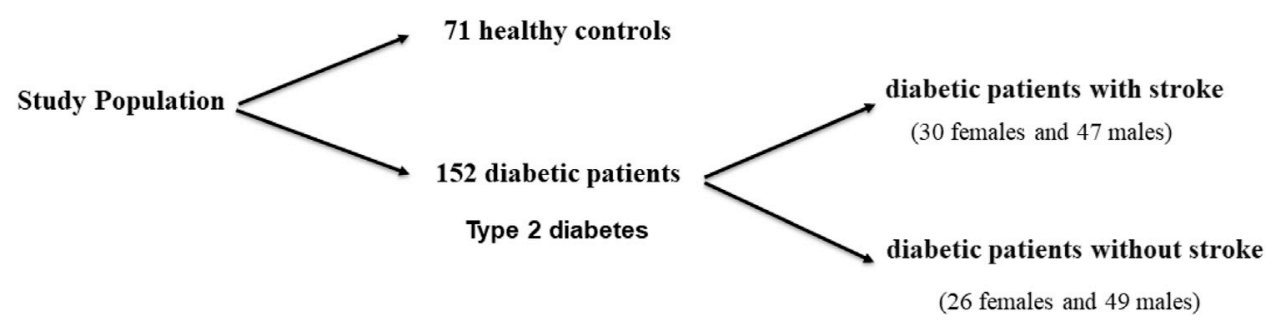

FIGURE 1 | Schematic diagram of the outline of the work performed in this study.

TABLE 1 | Baseline characteristics of the enrolled groups

$$
\text { Control }(n=71)
$$

Without stroke $(n=75)$

$$
\begin{gathered}
25(35.21 \%) \\
56(64.79 \%) \\
54.58 \pm 18.75 \\
29.37 \pm 1.82 \\
83.25 \pm 8.97 \\
111.85 \pm 10.24 \\
4.27 \pm 1.65 \\
18.74 \pm 3.89 \\
17.92 \pm 8.17 \\
24.71 \pm 8.74 \\
0.70 \pm 0.19 \\
12.13 \pm 3.24 \\
33.12 \pm 2.19 \\
28.11 \pm 2.31 \\
138.15 \pm 23.19 \\
49.57 \pm 19.87 \\
41.22 \pm 8.99 \\
65.13 \pm 9.13 \\
-
\end{gathered}
$$

$\mathrm{MCH}$

Cholesterol (mg/dl)

LDL (mg/dl)

$\mathrm{HDL}(\mathrm{mg} / \mathrm{dl})$

Triglycerides (mg/dl)

NIHSS

Disease duration (years)

$$
\begin{gathered}
26(34.67 \%) \\
49(65.33 \%) \\
53.19 \pm 17.78 \\
30.07 \pm 2.46 \\
154.58 \pm 28.11 \\
255.75 \pm 47.08 \\
7.87 \pm 2.34 \\
37.25 \pm 9.27 \\
32.25 \pm 6.87 \\
55.19 \pm 11.31 \\
2.72 \pm 0.34 \\
11.89 \pm 2.98 \\
33.09 \pm 2.01 \\
27.98 \pm 3.07 \\
168.25 \pm 18.52 \\
86.17 \pm 15.79 \\
35.12 \pm 7.58 \\
137.32 \pm 35.62 \\
- \\
13.35 \pm 1.87
\end{gathered}
$$

p-value ${ }^{a}$

With stroke $(n=77)$

$$
\begin{gathered}
30(38.96 \%) \\
47(61.04 \%) \\
57.08 \pm 16.31 \\
31.89 \pm 2.09 \\
185.41 \pm 40.85 \\
309.15 \pm 53.29 \\
9.07 \pm 3.12 \\
40.09 \pm 8.97 \\
35.51 \pm 8.71 \\
58.18 \pm 9.47 \\
3.09 \pm 0.17 \\
12.01 \pm 3.07 \\
32.97 \pm 2.05 \\
29.01 \pm 2.13 \\
198.16 \pm 34.08 \\
101.71 \pm 25.55 \\
30.09 \pm 8.88 \\
149.85 \pm 44.73 \\
11.38 \pm 5.12 \\
15.729 \pm 1.97
\end{gathered}
$$

p-value ${ }^{b}$

0.352
0.907
0.559
0.604
$\mathbf{0 . 0 4}^{\star}$
$\mathbf{0 . 0 4}^{\star}$
$\mathbf{0 . 0 2}^{\star}$
0.07
0.425
0.108
0.094
0.571
0.580
0.231
0.06
$\mathbf{0 . 0 3}$
0.064
0.091
-
0.09

Data are shown as the mean \pm ( $S D$, median (range), or $\mathrm{n}(\%)$.

BMI, body mass index; FBG, fasting blood glucose; 2hPP, 2 h post-prandial; HbA1c, glycated hemoglobin A1c; ALT, alanine transaminase; AST, aspartate transaminase; Hb, hemoglobin; MCV, mean corpuscular volume; MCH, mean corpuscular hemoglobin; LDL, low-density lipoprotein; HDL, high-density lipoprotein; NIHSS, National Institutes of Health Stroke Scale

*Significant at $\mathrm{p}<0.05$

${ }^{a}$ Comparison of diabetic patients (with and without stroke) versus the healthy control group

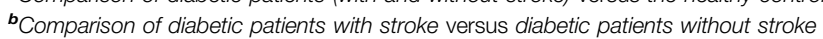

$\mathrm{RT}^{2}$ First Strand Kit (Qiagen, Germantown, MD, USA) according to the manufacturer's protocol for lncRNA expression analysis. Moreover, the miScript II RT Kit (Qiagen, Valencia, CA, US) was used for miRNA expression analysis in a $20-\mu$ l reverse transcription (RT) reaction according to the instructions in the pamphlet.

\section{LncRNA and miRNA Expression by Real-Time Quantitative PCR}

Real-time PCR amplification reactions were performed using the $\mathrm{RT}^{2}$ SYBR Green PCR Kit (Qiagen, Germantown, MD, USA) for the detection of lncRNA. However, the miScript SYBR Green PCR Kit (Qiagen, Valencia, CA, USA) was used in the quantification of miRNAs with the aid of the Rotor-Gene Q System (Qiagen).
The RefSeq accession no. of TUG1 was NR_002323.2 and that of LINC00657was NR_027451.1. GAPDH was used as an endogenous control for the evaluation of TUG1 and LINC00657 according to the manufacturer's instructions. Numerous studies have used GAPDH as an internal reference for serum lncRNAs (Duan et al., 2016; Shaker et al., 2019). The primer sequences of GAPDH were as follows: forward: $5^{\prime}$-CCCTTCATTGACCTCAAC TA-3'; reverse: 5-'TGGAAGATGGTGATGGGATT-3'.

Moreover, the catalog number of miR-9 was MS00010752 and that of miR-106a was MS00008393. SNORD68 was used as the internal reference for the evaluation of the gene expression levels of miR-9 and miR-106a. The catalog number of SNORD68 was MS00033712.

The PCR cycling program for the quantification of lncRNAs consists of an initial incubation at $95^{\circ} \mathrm{C}$ for $10 \mathrm{~min}$, followed by 40 
TABLE 2 | Expression levels of serum TUG1, LINC00657, miR-9, and miR-106a in all groups

\begin{tabular}{|c|c|c|c|}
\hline \multirow[t]{2}{*}{ Variables } & Diabetes without stroke $(n=75)$ & Diabetes with stroke $(n=77)$ & \multirow[t]{2}{*}{$p$-value } \\
\hline & \multicolumn{2}{|c|}{ Median (intraquartile range) } & \\
\hline TUG1 & $0.71(0.01-1.97)$ & $2.90(0.31-13.25)$ & $0.125^{\mathrm{a}}<\mathbf{0 . 0 0 1}^{\star \mathrm{b}, \mathrm{c}}$ \\
\hline LINC00657 & $3.18(0.87-20.98)$ & $11.85(0.50-53.85)$ & $0.001^{\star a}<0.001^{\star b, c}$ \\
\hline $\operatorname{miR}-9$ & $1.45(0.12-8.07)$ & $4.40(0.35-12.25)$ & $0.001^{\star a}<0.001^{\star b} 0.003^{\star c}$ \\
\hline $\mathrm{miR}-106 \mathrm{a}$ & $0.760(0.13-1.62)$ & $0.03(0.01-0.41)$ & 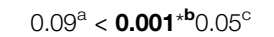 \\
\hline
\end{tabular}

Fold change levels represent non-coding RNA expression relative to controls that were calculated using $2^{-\Delta \Delta C T}$. Control fold change levels are equivalent to 1 . Data are expressed as the median and intraquartile range. Adjusted p-values for multiple comparisons of the studied groups were estimated using the Bonferroni correction method.

*Significant at $\mathrm{p}<0.017$

${ }^{a}$ Comparison of diabetes without stroke versus healthy controls

${ }^{\boldsymbol{b}}$ Comparison of diabetes with stroke versus healthy controls

${ }^{c}$ Comparison of diabetes with stroke versus diabetes without stroke

cycles at $95^{\circ} \mathrm{C}$ for $15 \mathrm{~s}$ and $60^{\circ} \mathrm{C}$ for $60 \mathrm{~s}$. That for the detection of miRNAs consists of $95^{\circ} \mathrm{C}$ for $30 \mathrm{~min}$, followed by 40 cycles at $94^{\circ} \mathrm{C}$ for $15 \mathrm{~s}, 55^{\circ} \mathrm{C}$ for $30 \mathrm{~s}$, and $70^{\circ} \mathrm{C}$ for $30 \mathrm{~s}$.

The relative expression levels of TUG1, LINC00657, miR-9, and miR-106a were calculated using $2^{-\Delta \Delta C t}$. Fold change (FC) values less than 1 indicated downregulation, while values more than 1 indicated upregulation of non-coding RNAs (Livak and Schmittgen, 2001). Control FC values were set as 1 .

\section{Statistical Analyses}

Statistical analysis was performed using Statistical Package for Social Sciences (SPSS) version 24. The mean, standard deviation (SD), median, and interquartile range (IQR) were utilized to represent quantitative data. A chi-square test was performed for categorical data. However, the Mann-Whitney $U$ test was used for continuous variables, which were presented as median (interquartile range). To determine the relation of the expressions of non-coding RNAs with the study parameters, Spearman's correlation was run. A multivariate stepwise logistic regression was constructed to identify the significant predictors of cerebral stroke among the four markers.

Analyses of the receiver operating characteristic (ROC) curves were conducted to determine the sensitivity and specificity of TUG1, LINC00657, miR-9, and miR-106a as predictors in differentiating between different groups. Statistical significance was considered at a $p$-value $<0.05$. Adjusted $p$-values for multiple comparisons of the studied groups were estimated using the Bonferroni correction method. The $p$-value (of 0.05) was divided by the number of comparisons, i.e., $3(0.05 / 3)$. Therefore, the test results were considered to be statistically significant at $p$-values $<0.017$.

\section{RESULTS}

\section{Clinical and Laboratory Features of the Enrolled Participants}

A total of 75 diabetic patients without stroke, 77 diabetic patients with stroke, and 71 healthy individuals were included in the current study.

There were marked differences between the total diabetic patients (with and without stroke) and the healthy group regarding $\mathrm{FBG}$, 2hPP, HbAIc, alanine transaminase (ALT), aspartate transaminase (AST), urea, creatinine, cholesterol, LDL, high-density lipoprotein (HDL), and triglycerides (all $p<$ 0.05). However, no significant differences in age, sex, and other clinical and laboratory data were observed between all diabetic patients and control participants $(p>0.05)$ (Table 1). Moreover, there were significant differences concerning FBG, 2hPP, HbAIc, and LDL when comparing diabetic patients with stroke to those without stroke (all $p<0.05$ ). On the other hand, there were no marked differences regarding age, sex, and all other data when comparing diabetic patients with stroke to those without stroke $(p>0.05)$ (Table 1).

\section{Comparison of the Serum Expression Levels of TUG1, LINC00657, miR-9, and miR-106a in the Different Studied Groups}

As clarified in Table 2, the serum expression levels of LINC00657 and miR-9 were increased significantly in diabetic patients without stroke when compared to healthy individuals ( $p=$ 0.001 for LINC00657 and miR-9).

We next compared the expressions of TUG1, LINC00657, miR-9, and miR-106a in the sera of diabetic patients with stroke relative to healthy controls. The results showed significant upregulation of TUG1, LINC00657, and miR-9 ( $p<0.001$ for TUG1, LINC00657, and miR-9). In contrast, the level of miR-106a in serum was markedly decreased in diabetic patients who had stroke relative to the control subjects $(p<0.001)$.

Furthermore, we revealed a marked elevation of the serum expressions of TUG1, LINC00657, and miR-9 in diabetic patients with stroke relative to those without stroke $(p<0.001$ for TUG1 and LINC00657; $p=0.003$ for miR-9). Meanwhile, a nonsignificant decrease of miR-106a was detected between diabetic patients with stroke and those without stroke $(p=0.05)$.

\section{Correlation of TUG1, LINC00657, miR-9, and miR-106a With Stroke Severity and Clinical Characteristics}

NIHSS scoring was performed to evaluate stroke severity. We used Spearman's analysis to assess the correlation between the 
TABLE 3 | Correlation between the expression levels of serum non-coding RNAs and clinical parameters in diabetic patients with stroke

\begin{tabular}{|c|c|c|c|c|}
\hline Variables & TUG1 & LINC00657 & $\operatorname{miR}-9$ & miR-106a \\
\hline Disease duration & $0.754(<0.001)^{\star}$ & $0.720(<0.001)^{\star}$ & $0.675(<0.001)^{\star}$ & $-0.600(<0.001)^{\star}$ \\
\hline NIHSS & $0.802(<0.001)^{\star}$ & $0.709(<0.001)^{\star}$ & $0.681(<0.001)^{\star}$ & $-0.569(0.001)^{\star}$ \\
\hline Age & 0.097 (0.821) & $-0.074(0.893)$ & 0.107 (0.275) & $-0.197(0.104)$ \\
\hline BMl & 0.099 (0.752) & $0.055(0.708)$ & $0.122(0.564)$ & $-0.189(0.262)$ \\
\hline FBG & 0.017 (0.920) & $0.213(0.206)$ & $0.109(0.523)$ & $0.269(0.107)$ \\
\hline 2hPP & $0.094(0.578)$ & $0.172(0.310)$ & $0.102(0.548)$ & $0.114(0.501)$ \\
\hline $\mathrm{HbA1c}$ & $-0.014(0.779)$ & $0.098(0.587)$ & 0.073 (0.669) & $-0.107(0.527)$ \\
\hline AST & 0.034 (0.839) & 0.098 (0.565) & $0.013(0.941)$ & 0.277 (0.096) \\
\hline ALT & $-0.123(0.467)$ & $-0.187(0.267)$ & $-0.085(0.618)$ & $-0.264(0.114)$ \\
\hline Urea & $-0.076(0.653)$ & $-0.146(0.388)$ & $-0.123(0.467)$ & $-0.138(0.416)$ \\
\hline Creatinine & $-0.127(0.454)$ & $-0.115(0.499)$ & $-0.125(0.460)$ & 0.069 (0.686) \\
\hline Cholesterol & $0.101(0.552)$ & $0.136(0.421)$ & 0.187 (0.268) & $-0.131(0.441)$ \\
\hline LDL & 0.037 (0.830) & $-0.053(0.755)$ & -0.052 (0.684) & $0.171(0.244)$ \\
\hline $\mathrm{HDL}$ & $-0.012(0.890)$ & $0.113(0.474)$ & $0.124(0.466)$ & $-0.165(0.328)$ \\
\hline Triglycerides & $0.051(0.765)$ & $0.011(0.872)$ & 0.029 (0.865) & $-0.097(0.524)$ \\
\hline
\end{tabular}

BMl, body mass index; FBG, fasting blood glucose; 2hPP, 2 h post-prandial; HbA1c, glycated hemoglobin A1c; ALT, alanine transaminase; AST, aspartate transaminase; Hb, hemoglobin; LDL, low-density lipoprotein; HDL, high-density lipoprotein; NIHSS, National Institutes of Health Stroke Scale

*Significant at $\mathrm{p}<0.05$

aforementioned ncRNAs and stroke severity, as well as clinical and laboratory data.

As demonstrated in Table 3, TUG1, LINC00657, and miR-9 were positively correlated with NIHSS $(r=0.802, p<0.001 ; r=$ $0.709, p<0.001$; and $r=0.681, p<0.001$, respectively). At the same time, a negative correlation was shown between miR-106a and NIHSS $(r=-0.569, p=0.001)$. In addition, TUG1, LINC00657, and miR-9 were positively correlated with disease duration $(r=0.754, p<0.001 ; r=0.720, p<0.001$; and $r=0.675$, $p<0.001$, respectively). On the other hand, a negative correlation was observed between miR-106a and years since the occurrence of DM $(r=-0.600, p<0.001)$.

However, no significant correlation was detected between TUG1, LINC00657, miR-9, and miR-106a and the laboratory parameters in the present study (all $p>0.05)$.

\section{Correlation of TUG1 With miR-9 and of LINC00657 With miR-106a}

Interestingly, the current results reported a negative correlation between LINC00657 and miR-106a $(r=-0.507, p=0.002)$ in diabetic patients with stroke. However, no significant correlation was shown between the serum levels of TUG1 and miR-9 $(r=$ $0.251, p=0.10)$.

\section{ROC Analysis to Determine the Diagnostic Performance of Serum TUG1, LINC00657, miR-9, and miR-106a in Distinguishing Diabetic Patients With Stroke From Control Subjects}

An ROC curve was assembled to estimate the diagnostic value of TUG1, LINC00657, miR-9, and miR-106a as novel biomarkers for DM with stroke relative to healthy subjects. For TUG1, the AUC was 0.758 (95\% CI $=0.669-0.846, p<0.001)$, with a sensitivity of $48.50 \%$ and a specificity of $100 \%$. Moreover, the AUC of LINC00657 was
$0.892(95 \% \mathrm{CI}=0.834-0.950, p<0.001)$, with a sensitivity of $73.50 \%$ and a specificity of $100 \%$. Also, the AUC of miR-9 was 0.755 (95\% CI $=0.677-0.834, p<0.001$ ), with a sensitivity of $39.5 \%$ and a specificity of $100 \%$. Regarding miR-106a, its AUC was 0.674 (95\% CI = $0.583-0.765, p<0.001$ ), and the sensitivity and specificity were $38.4 \%$ and $100 \%$, respectively. On the other hand, the AUC of LDL was 0.979 (95\% CI $=0.962-0.996, p<0.001)$, with sensitivity of $87.5 \%$ and specificity of $45.8 \%$ (Table 4 and Figure 2 ).

ROC curve analysis revealed that serum TUG1, LINC00657, miR9, and miR-106a have good value as prognostic markers in discriminating diabetic patients with stroke from those without stroke.

The current results demonstrated that using TUG1 to diagnose diabetes with stroke yielded an AUC of 0.954 (95\% CI $=0.915-0.994$, $p<0.001$ ), with a sensitivity of $87.9 \%$ and a specificity of $98.5 \%$. In addition, the AUC value for LINC00657 was 0.902 (95\% CI = 0.847-0.957, $p<0.001$ ), with a sensitivity of $35.1 \%$ and a specificity of $98.5 \%$. Also, miR-9 had an AUC of 0.661 (95\% CI = $0.571-0.752, p<0.001)$ and sensitivity and specificity values of $39.0 \%$ and $93.5 \%$, respectively. For miR-106a, the AUC was 0.747 (95\% CI = $0.661-0.832, p=0.01)$, with a sensitivity of $39.0 \%$ and a specificity of $100 \%$, while the AUC of LDL was $0.736(95 \% \mathrm{CI}=0.654-0.819, p<$ 0.001 ) and the sensitivity and specificity were $20 \%$ and $90.2 \%$, respectively (Table 5 and Figure 3).

\section{Multiple Logistic Regression Analysis}

Multivariate regression analysis (considering NIHSS as the dependent variable) confirmed that TUG1 and LINC00657 were independent predictors for diabetes with stroke $(p=0.04$ and $p=0.01$, respectively) (Table 6).

\section{DISCUSSION}

Type 2 diabetes mellitus (T2DM) has emerged as a cause of serious concern worldwide and has been established as a risk factor for ischemic stroke (Nakagami et al., 2005). It is important 
TABLE 4 | Receiver operating characteristics (ROC) curve analysis using serum TUG1, LINC00657, miR-9, miR-106a, and LDL for discriminating diabetic patients with stroke from control subjects

\begin{tabular}{|c|c|c|c|c|c|}
\hline Variable & AUC $(95 \% \mathrm{Cl})$ & $p$-value & Sensitivity (\%) & Specificity (\%) & Total accuracy \\
\hline TUG1 & 0.758 (0.669-0.846) & $<0.001^{\star}$ & 48.50 & 100 & 74.25 \\
\hline LINC00657 & $0.892(0.834-0.950)$ & $<0.001^{*}$ & 73.50 & 100 & 86.75 \\
\hline miR-9 & $0.755(0.677-0.834)$ & $<0.001^{\star}$ & 39.5 & 100 & 69.75 \\
\hline miR-106a & $0.674(0.583-0.765)$ & $<0.001^{\star}$ & 38.4 & 100 & 69.20 \\
\hline LDL & $0.979(0.962-0.996)$ & $<0.001^{*}$ & 87.5 & 45.8 & 66.65 \\
\hline
\end{tabular}

AUC, area under the curve; $\mathrm{Cl}$, confidence interval; LDL, low-density lipoprotein

*Significant at $\mathrm{p}<0.05$.

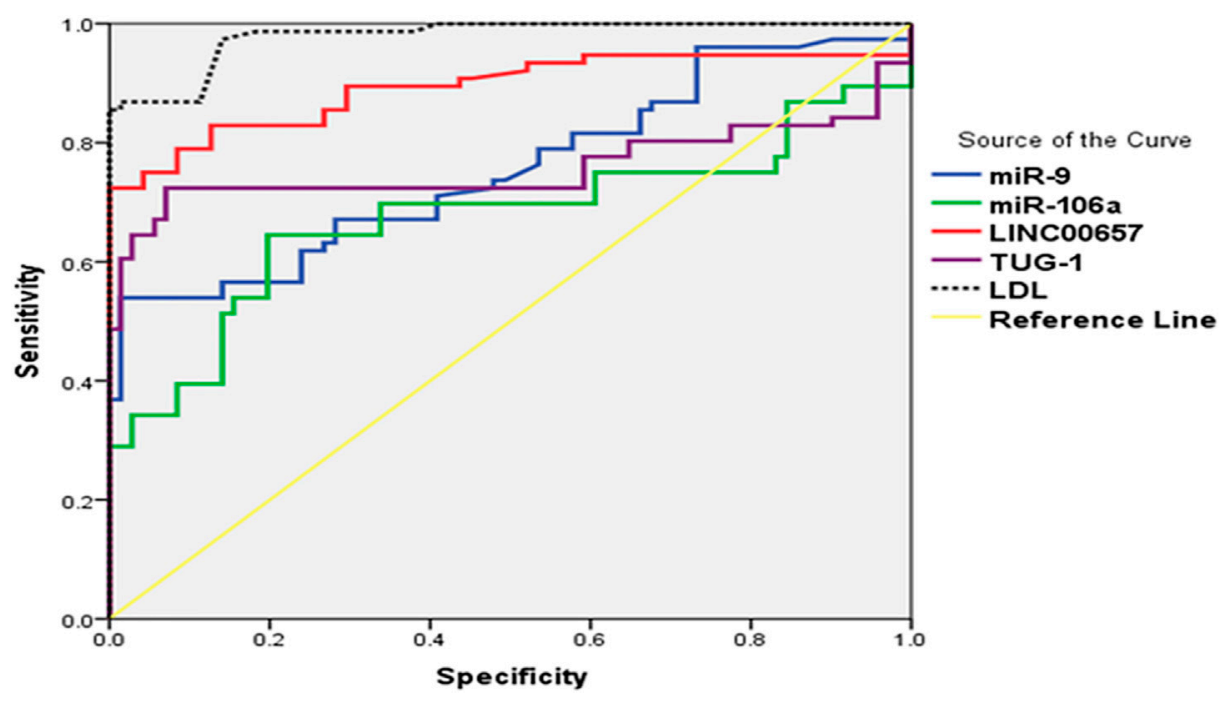

FIGURE 2 | (ROC) curve analysis of serum TUG1, LINC00657, miR-9, and miR-106a for distinguishing diabetic patients without stroke from control subjects.

to discover new sensitive and easily detected biomarkers for the diagnosis and prognosis of T2DM and its related complications.

Recently, it has been shown that non-coding RNAs (including lncRNAs and microRNAs) may be used as probable biomarkers for T2DM and its associated complications due to their stability and differential expression in a variety of body fluids, such as plasma and serum (Mastropasqua et al., 2014; Shaker et al., 2019). However, no previous reports have investigated the role of TUG1, LINC00657, miR-9, and miR-106a in stroke associated with DM. Thus, in this article, we assessed the serum expression levels of TUG1, LINC00657, miR-9, and miR-106a in diabetic patients with and without stroke.

We observed low TUG1 and miR-106a and significantly high LINC00657 and miR-9 expression levels in the serum of diabetic patients without stroke compared to control participants. At the same time, we verified marked increases of serum TUG1, LINC00657, and miR-9 and a marked decrease of serum miR106a in diabetic patients who had stroke relative to those without stroke. Previous studies reported that the expression of TUG1 was decreased in rats with diabetes and in mesangial cells induced with high-level glucose through inhibition of the PI3K/AKT pathway (Zang et al., 2019). Furthermore, Wang et al. showed that TUG1 was downregulated in NRK-52E cells (high-glucose- stimulated) in mice via targeting miR-21 (Wang et al., 2019). Similarly, Li et al. documented a low expression level of TUG1 in high-glucose-stimulated podocytes by hindering the expression of miR-27a-3p (Li et al., 2019).

Our results regarding the upregulation of TUG1 in diabetic patients who had stroke are in line with recent studies showing that TUG1 was overexpressed in ischemic stroke by regulating miR-9 and decreasing Bcl-2-like 11 protein [25]. Also, in atherosclerosis, the elevated expression level of TUG1 increased endothelial cell apoptosis through miR-26a sponging (Chen et al., 2016). Similarly, many recent studies have discussed the role of TUG1 in atherosclerosis. For example, Li et al. found that TUG1, via regulating the miR-21/PTEN axis, increased the proliferation of vascular smooth muscle (Li et al., 2018). In addition, Yan et al. documented the role of TUG1 in the migration and proliferation of endothelial cells by the Wnt pathway (Yan et al., 2018). Moreover, Zhang et al. reported that TUG1 knockdown ameliorated atherosclerotic lesion and inhibited inflammation and hyperlipidemia via the upregulation of fibroblast growth factor 1 (Zhang et al., 2018). Besides, Yang et al. noted an increased expression level of TUG1 in ischemic heart exposed to oxidative stress via increasing cardiomyocyte apoptosis (Yang et al., 2019). 
TABLE 5 | Receiver operating characteristics (ROC) curve analysis using serum TUG1, LINC00657, miR-9, and miR-106a for discriminating diabetic patients with stroke from diabetic patients without stroke

\begin{tabular}{|c|c|c|c|c|c|}
\hline Variable & AUC $(95 \% \mathrm{Cl})$ & $p$-value & Sensitivity (\%) & Specificity (\%) & Total accuracy \\
\hline TUG1 & $0.954(0.915-0.994)$ & $<0.001^{\star}$ & 87.9 & 98.5 & 93.2 \\
\hline LINC00657 & $0.902(0.847-0.957)$ & $<0.001^{*}$ & 35.1 & 98.5 & 66.8 \\
\hline miR-9 & $0.661(0.571-0.752)$ & $0.001^{\star}$ & 39.0 & 93.5 & 51.25 \\
\hline miR-106a & $0.747(0.661-0.832)$ & $<0.001^{\star}$ & 39.0 & 100 & 69.5 \\
\hline LDL & $0.736(0.654-0.819)$ & $<0.001^{\star}$ & 20 & 90.2 & 55.1 \\
\hline
\end{tabular}

AUC, area under the curve; Cl, confidence interval; LDL, low-density lipoprotein

*Significant at $\mathrm{p}<0.05$.

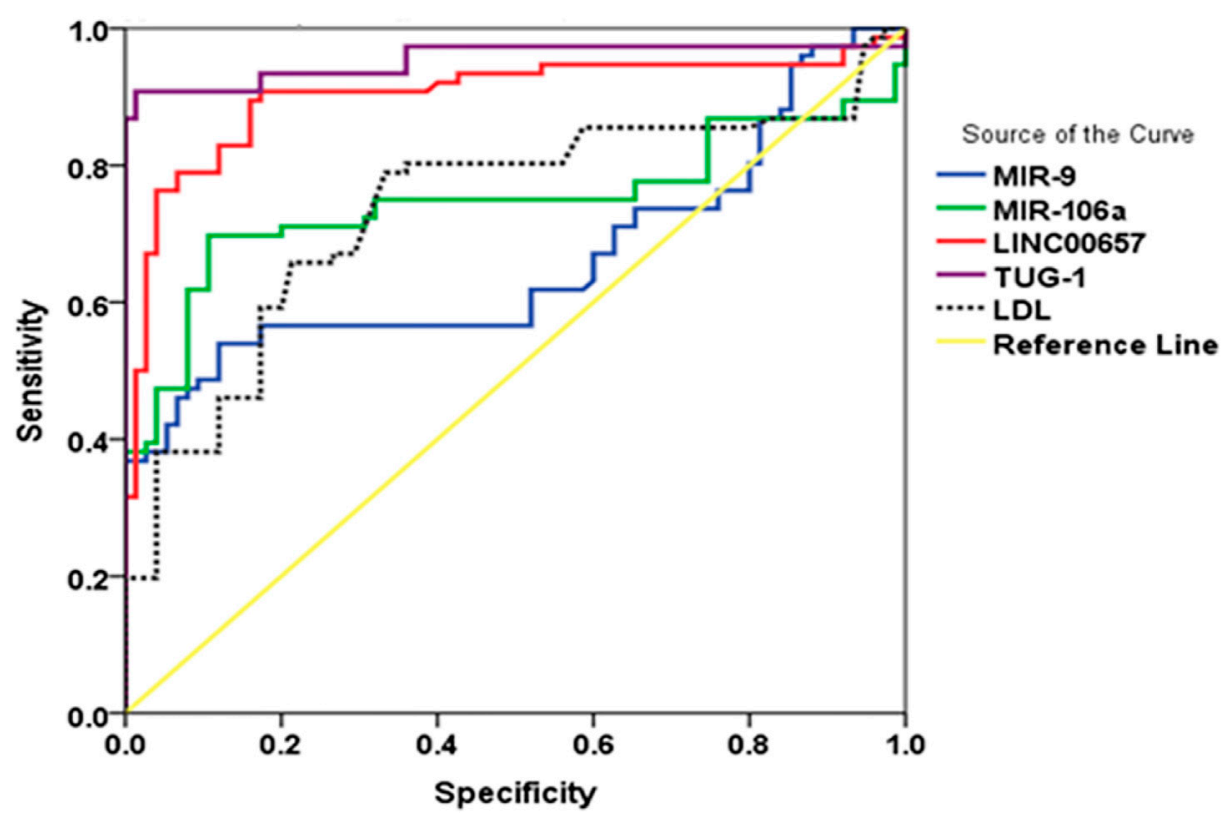

FIGURE 3 | (ROC) curve analysis of serum TUG1, LINC00657, miR-9, and miR-106a for discriminating diabetic patients with stroke from those without stroke.

However, there are no reports on the relation between TUG1 and stoke associated with DM.

Regarding LINC00657 (NORAD), our results are in line with a study which found that LINC00657, which is expressed in vascular endothelial cells, induced angiogenesis during atherosclerosis through the upregulation of VEGF, MMP-2, and MMP-9 (Wan et al., 2020). Also, Michalik et al. revealed that LINC00657 was markedly elevated during hypoxia (Michalik et al., 2014). Of note is that Bao et al. reported that oxidized LDL (oxLDL) treatment, which promotes oxidative stress and is implicated in atherosclerosis, resulted in the overexpression of LINC00657 (Bao et al., 2018b). Since hypoxia and atherosclerosis are predisposing factors of ischemic stroke, we therefore assumed that LINC00657 might contribute to the pathogenesis of stroke.

In the current research, we assessed the expression level of miR-9, which is a target gene of TUG1 and miR-106a, which are target genes of LINC00657. It was revealed in previous studies that the serum expression level of miR-9 increased significantly in T2DM (Kong et al., 2011), which is in accordance with our results. Furthermore, miR-9 was found to decrease insulin secretion via targeting syntaxin-binding protein 1, Onecut 2, and sirtuin 1 (Sirt1) (Plaisance et al., 2006; Ramachandran et al., 2011; Hu et al., 2018). However, Jiménez-Lucena et al. reported a low plasma level of miR-9 in patients at risk of T2DM (Jiménez-Lucena et al., 2018).

More importantly, previous studies also demonstrated the role of miR-9 in ischemic stroke, such as Ji et al. who found that miR-9 was upregulated in the serum exosomes of patients with acute ischemic stroke and was strongly associated with interleukin 6 (IL-6) production (Ji et al., 2016). In addition, the serum expression level of miR-9 was verified to be elevated significantly in acute ischemic stroke patients and was positively correlated with inflammatory markers, infarct volume, and the NIHSS score (Ji et al., 2016). Besides, another study has considered miR-9 to be a new biomarker of neurotoxicity and neural damage (Xue et al., 2018). At the same time, an increasing number of studies have explained the role of miR-9 in neuronal apoptosis after ischemic stroke (Wei et al., 2016). 
TABLE 6 | Multiple logistic regression analysis

\begin{tabular}{lccccc} 
& \multicolumn{1}{c}{$\boldsymbol{B}$} & SE & $\boldsymbol{p}$-value & \multicolumn{2}{c}{$\mathbf{9 5 \%}$ CI for $\boldsymbol{B}$} \\
\cline { 5 - 6 } & & & & Lower & Upper \\
\hline TUG1 & -5.980 & 1.071 & $\mathbf{0 . 0 2}^{\star}$ & 2.82 & 11.2 \\
LINC00657 & -19.248 & 2.09 & $\mathbf{0 . 0 3}^{\star}$ & 1.02 & 5.54 \\
miR-9 & -0.633 & 0.115 & 0.124 & 0.91 & 2.11 \\
miR-106a & 4.135 & 3.294 & 0.247 & 0.44 & 5.52 \\
LDL & 0.012 & 0.007 & 0.078 & -0.001 & 0.025 \\
Disease duration & 0.243 & 0.082 & $\mathbf{0 . 0 0 4}$ & 0.080 & 0.406 \\
FBG & -0.005 & 0.017 & 0.786 & -0.038 & 0.029 \\
HbA1c & -0.311 & -0.196 & 0.117 & -0.702 & 0.080 \\
Constant & 9.464 & 3.688 & 0.013 & 2.100 & 16.828
\end{tabular}

$\mathrm{Cl}$, confidence interval; LDL, low-density lipoprotein; FBG, fasting blood glucose; $2 \mathrm{hPP}$,

$2 \mathrm{~h}$ post-prandial; HbA1c, glycated hemoglobin A1C

*Significant at $\mathrm{p}<0.05$

Concerning miR-106a, our findings are in line with the study by $\mathrm{Wu}$ et al., which determined that miR-106a was decreased in diabetic peripheral neuropathy via the regulation of $12 / 15$ lipoxygenase of oxidative/nitrative stress ( $\mathrm{Wu}$ et al., 2017). Previous findings demonstrated the role of miR-106a in numerous risk factors of ischemic stroke. For example, under oxidative stress, miR-106-5p was documented to be decreased, causing premature senescence by suppressing the G1/S-phase transition of the cell cycle through modulating the expression of E2F1 (Tai et al., 2020). Similarly, increased levels of reactive oxygen species (ROS) resulted to the decreased expression of miR-106a (Wang et al., 2010). On the other hand, elevated levels of miR-106a prevented oxidative stress injury and inflammation in hepatic mouse with gestational hypertension (Wang $\mathrm{Z}$. et al., 2019), resulting to repression of the expressions of HIF1- $\alpha$ and VEGF in diabetic retina (Ling et al., 2013). In addition, miR-106a has been associated with macrophage activation, suggesting its involvement in inflammation (Zhu et al., 2013).

In the present work, it was interesting to find a negative correlation between LINC00657 and miR-106a in diabetic patients who had stroke. A number of recent studies have hypothesized that lncRNAs could affect the progression of diseases through regulating miRNAs. It was reported that LINC00657 could influence tumorigenesis in hepatocellular carcinoma by regulating miR-106a (Hu et al., 2017).

Notably, an ROC curve was constructed in our study. The results implied that serum TUG1, LINC00657, miR-9, and miR106a could discriminate diabetic patients without stroke from healthy subjects. More importantly, the aforementioned non-

\section{REFERENCES}

Bao, M.-H., Szeto, V., Yang, B. B., Zhu, S.-Z., Sun, H.-S., and Feng, Z.-P. (2018a). Long Non-coding RNAs in Ischemic Stroke. Cell Death Dis 9, 281. doi:10.1038/ s41419-018-0282-x

Bao, M. H., Li, G. Y., Huang, X. S., Tang, L., Dong, L. P., and Li, J. M. (2018b). Long Noncoding RNA LINC00657 Acting as a miR-590-3p Sponge to Facilitate Low coding RNAs may be used to differentiate diabetic patients with stroke from those without stroke.

Some limitations of this work should be addressed. First is the relatively small sample size. Therefore, further works with larger sample sizes in various populations are needed. Moreover, further experiments are necessary to explain the detailed mechanisms of the role of these non-coding RNAs in diabetic patients with and without stroke.

\section{CONCLUSION}

The current study, for the first time, revealed that serum TUG1, LINC00657, miR-9, and miR-106a may serve as novel potential indicators of stroke associated with diabetes and correlated significantly with NIHSS. Furthermore, they might be used as new targets of treatment for diabetic patients with stroke.

\section{DATA AVAILABILITY STATEMENT}

The datasets presented in this article are not readily available due to patient confidentiality. Requests to access the datasets should be directed to dr.omayma@yahoo.com.

\section{ETHICS STATEMENT}

The studies involving human participants were reviewed and approved by the Ethics Committee of the Faculty of Medicine, Fayoum University. The patients/participants provided written informed consent to participate in this study.

\section{AUTHOR CONTRIBUTIONS}

$\mathrm{MM}, \mathrm{AE}$, and TA performed the patient examination and treatments. OS, RM, OA, NKA, and $\mathrm{NH}$ performed the biochemical assays. AK and NAA interpreted the data. OA and RM were major contributors to the writing of the manuscript. All authors have read and approved the final manuscript.

\section{ACKNOWLEDGMENTS}

We thank all the medical and paramedical staff who helped in the achievement of this work.

Concentration Oxidized Low-Density Lipoprotein-Induced Angiogenesis. Mol. Pharmacol. 93, 368-375. doi:10.1124/mol.117.110650

Chen, C., Cheng, G., Yang, X., Li, C., Shi, R., and Zhao, N. (2016). Tanshinol Suppresses Endothelial Cells Apoptosis in Mice with Atherosclerosis via IncRNA TUG1 Up-Regulating the Expression of miR-26a. Am. J. Transl. Res. 8, 2981-2991.

Chen, S., Wang, M., Yang, H., Mao, L., He, Q., Jin, H., et al. (2017). LncRNA TUG1 Sponges microRNA-9 to Promote Neurons Apoptosis by Up-Regulated Bcl2111 
under Ischemia. Biochem. Biophys. Res. Commun. 485, 167-173. doi:10.1016/j. bbrc.2017.02.043

Duan, W., Du, L., Jiang, X., Wang, R., Yan, S., Xie, Y., et al. (2016). Identification of a Serum Circulating IncRNA Panel for the Diagnosis and Recurrence Prediction of Bladder Cancer. Oncotarget 7, 78850-78858. doi:10.18632/oncotarget.12880

Ferreiro, J. L., Gómez-Hospital, J. A., and Angiolillo, D. J. (2010). Review Article: Platelet Abnormalities in Diabetes Mellitus. Diabetes Vasc. Dis. Res. 7, 251-259. doi:10.1177/1479164110383994

Hu, B., Cai, H., Zheng, R., Yang, S., ZhouZand Tu, J. (2017). Long Non-coding RNA 657 Suppresses Hepatocellular Carcinoma Cell Growth by Acting as a Molecular Sponge of miR-106a-5p to Regulate PTEN Expression. Int. J. Biochem. Cel Biol 92, 34-42. doi:10.1016/j.biocel.2017.09.008

Hu, D., Wang, Y., Zhang, H., and Kong, D. (2018). Identification of miR-9 as a Negative Factor of Insulin Secretion from Beta Cells. J. Physiol. Biochem. 74, 291-299. doi:10.1007/s13105-018-0615-3

Iyengar, B. R., Choudhary, A., Sarangdhar, M. A., Venkatesh, K. V., Gadgil, C. J., and Pillai, B. (2014). Non-coding RNA Interact to Regulate Neuronal Development and Function. Front. Cel. Neurosci. 8, 47. doi:10.3389/fncel. 2014.00047

Ji, Q., Ji, Y., Peng, J., Zhou, X., Chen, X., Zhao, H., et al. (2016). Increased Brainspecific MiR-9 and MiR-124 in the Serum Exosomes of Acute Ischemic Stroke Patients. PLoS ONE 11, e0163645. doi:10.1371/journal.pone.0163645

Jiménez-Lucena, R., Rangel-Zúñiga, O. A., Alcalá-Díaz, J. F., López-Moreno, J., Roncero-Ramos, I., Molina-Abril, H., et al. (2018). Circulating miRNAs as Predictive Biomarkers of Type 2 Diabetes Mellitus Development in Coronary Heart Disease Patients from the CORDIOPREV Study. Mol. Ther. - Nucleic Acids 12, 146-157. doi:10.1016/j.omtn.2018.05.002

Khoshnam, S. E., Winlow, W., Farbood, Y., Moghaddamand, H. F., and Farzaneh, M. (2017). Emerging Roles of microRNAs in Ischemic Stroke: As Possible Therapeutic Agents. J. Stroke 19, 166-187. doi:10.5853/jos.2016.01368

Kitagawa, M., Kitagawa, K., Kotake, Y., Niida, H., and Ohhata, T. (2013). Cell Cycle Regulation by Long Non-coding RNAs. Cell. Mol. Life Sci. 70, 4785-4794. doi:10.1007/s00018-013-1423-0

Kong, L., Zhu, J., Han, W., Jiang, X., Xu, M., Zhao, Y., et al. (2011). Significance of Serum microRNAs in Pre-diabetes and Newly Diagnosed Type 2 Diabetes: a Clinical Study. Acta Diabetol. 48, 61-69. doi:10.1007/s00592-010-0226-0

Koutsis, G., Siasos, G., and Spengos, K. (2013). The Emerging Role of microRNA in Stroke. Curr. Top. Med. Chem. 13, 1573-1588. doi:10.2174/ 15680266113139990106

Li, F. P., Lin, D. Q., and Gao, L. Y. (2018). LncRNA TUG1 Promotes Proliferation of Vascular Smooth Muscle Cell and Atherosclerosis through Regulating miRNA-21/PTEN axis. Eur. Rev. Med. Pharmacol. Sci. 22, 7439-7447. doi:10.26355/eurrev_201811_16284

Li, J. H., Liu, S., Zhou, H., Qu, L. H., and Yang, J. H. (2014). starBase v2.0: Decoding miRNA-ceRNA, miRNA-ncRNA and Protein-RNA Interaction Networks from Large-Scale CLIP-Seq Data. Nucleic Acids Res. 42 (Database issue), D92-D97. doi:10.1093/nar/gkt1248

Li, Y., Huang, D., Zheng, L., Cao, H., Gao, Y., Yang, Y., et al. (2019). Retracted Article: Long Non-coding RNA TUG1 Alleviates High Glucose Induced Podocyte Inflammation, Fibrosis and Apoptosis in Diabetic Nephropathy via Targeting the miR-27a-3p/E2F3 axis. RSC Adv. 9, 37620-37629. doi:10. 1039/c9ra06136c

Ling, S., Birnbaum, Y., Nanhwan, M. K., Thomas, B., Bajaj, M., and Ye, Y. (2013). MicroRNA-dependent Cross-Talk between VEGF and HIFla in the Diabetic Retina. Cell Signal. 25, 2840-2847. doi:10.1016/j.cellsig.2013.08.039

Liu, H., Li, J., Koirala, P., Ding, X., Chen, B., Wang, Y., et al. (2016). Long Noncoding RNAs as Prognostic Markers in Human Breast Cancer. Oncotarget 7, 20584-20596. doi:10.18632/oncotarget.7828

Liu, S., Zou, L., Xie, J., Xie, W., Wen, S., Xie, Q., et al. (2016). LncRNA NONRATT021972 siRNA Regulates Neuropathic Pain Behaviors in Type 2 Diabetic Rats through the P2X7 Receptor in Dorsal Root Ganglia. Mol. Brain 9, 44. doi:10.1186/s13041-016-0226-2

Livak, K. J., and Schmittgen, T. D. (2001). Analysis of Relative Gene Expression Data Using Real-Time Quantitative PCR and the $2-\Delta \Delta$ CT Method. Methods 25, 402-408. doi:10.1006/meth.2001.1262

Long, J., Badal, S. S., Ye, Z., Wang, Y., Ayanga, B. A., Galvan, D. L., et al. (2016). Long Noncoding RNA Tug1 Regulates Mitochondrial Bioenergetics in Diabetic Nephropathy. J. Clin. Invest. 126, 4205-4218. doi:10.1172/JCI87927
Mastropasqua, R., Toto, L., Cipollone, F., Santovito, D., Carpineto, P., and Mastropasqua, L. (2014). Role of microRNAs in the Modulation of Diabetic Retinopathy. Prog. Retin. Eye Res. 43, 92-107. doi:10.1016/j.preteyeres.2014. 07.003

Meng, S., Cao, J. T., Zhang, B., Zhou, Q., Shen, C. X., and Wang, C. Q. (2012). Downregulation of microRNA-126 in Endothelial Progenitor Cells from Diabetes Patients, Impairs Their Functional Properties, via Target Gene Spred-1. J. Mol. Cel Cardiol 53, 64-72. doi:10.1016/j.yjmcc.2012.04.003

Mercer, T. R., and Mattick, J. S. (2013). Structure and Function of Long Noncoding RNAs in Epigenetic Regulation. Nat. Struct. Mol. Biol. 20, 300-307. doi:10. 1038/nsmb. 2480

Michalik, K. M., You, X., Manavski, Y., Doddaballapur, A., Zörnig, M., Braun, T., et al. (2014). Long Noncoding RNA MALAT1 Regulates Endothelial Cell Function and Vessel Growth. Circ. Res. 114, 1389-1397. doi:10.1161/ circresaha.114.303265

Nakagami, H., Kaneda, Y., Ogihara, T., and Morishita, R. (2005). Endothelial Dysfunction in Hyperglycemia as a Trigger of Atherosclerosis. Cdr 1, 59-63. doi:10.2174/1573399052952550

Pinsker, J. E., Shank, T., Dassau, E., and Kerr, D. (2015). Comment on American Diabetes Association. Approaches to glycemic treatment. Sec. 7. In Standards of Medical Care in Diabetes-2015. Diabetes Care 2015; 38(Suppl. 1):S41-S48. Diabetes Care 38 (10), e174. doi:10.2337/dc15-0839

Plaisance, V., Abderrahmani, A., Perret-Menoud, V., Jacquemin, P., LemaigreandRegazzi, F. R., and Regazzi, R. (2006). MicroRNA-9 Controls the Expression of Granuphilin/Slp4 and the Secretory Response of InsulinProducing Cells. J. Biol. Chem. 281, 26932-26942. doi:10.1074/jbc.M601225200

Ramachandran, D., Roy, U., Garg, S., Ghosh, S., PathakandKolthur-Seetharam, S. U., and Kolthur-Seetharam, U. (2011). Sirt1 and Mir-9 Expression Is Regulated during Glucose-Stimulated Insulin Secretion in Pancreatic $\beta$-islets. FEBSJ 278, 1167-1174. doi:10.1111/j.1742-4658.2011.08042.x

Shaker, O. G., Abdelaleem, O. O., Mahmoud, R. H., Abdelghaffar, N. K., Ahmed, T. I., Said, O. M., et al. (2019). Diagnostic and Prognostic Role of Serum miR-20b, miR-17-3p, HOTAIR, and MALAT1 in Diabetic Retinopathy. IUBMB Life 71, 310-320. doi:10.1002/iub.1970

Tai, L., Huang, C. J., Choo, K. B., Cheong, S. K., and Kamarul, T. (2020). Oxidative Stress Down-Regulates MiR-20b-5p, MiR-106a-5p and E2F1 Expression to Suppress the G1/S Transition of the Cell Cycle in Multipotent Stromal Cells. Int. J. Med. Sci. 17, 457-470. doi:10.7150/ijms.38832

Wan, W., Wan, W., Long, Y., Li, Q., Jin, X., Wan, G., et al. (2020). RETRACTED ATICLE: Physcion 8-O- $\beta$-Glucopyranoside Exerts Protective Roles in High Glucose-Induced Diabetic Retinopathy via Regulating lncRNA NORAD/miR125/STAT3 Signalling. Artif. Cell Nanomedicine, Biotechnol. 48, 463-472. doi:10.1080/21691401.2019.1709861

Wang, W.-Y., Wang, Y.-F., Ma, P., Xu, T.-P., and Shu, Y.-Q. (2017). Taurineupregulated Gene 1: A Vital Long Non-coding RNA Associated with Cancer in Humans. Mol. Med. Rep. 16, 6467-6471. doi:10.3892/mmr.2017.7472

Wang, F., Gao, X., Zhang, R., Zhao, P., Sun, Y., and Li, C. (2019). LncRNA TUG1 Ameliorates Diabetic Nephropathy by Inhibiting miR-21 to Promote TIMP3Expression. Int. J. Clin. Exp. Pathol. 12, 717-729.

Wang, Z., Bao, X., Song, L., Tian, Y., and Sun, P. (2019). Role of miR-106-mediated Mitogen-activated Protein Kinase Signaling Pathway in Oxidative Stress Injury and Inflammatory Infiltration in the Liver of the Mouse with Gestational Hypertension. J. Cel Biochem 122, 958-968. doi:10.1002/jcb.29552

Wang, Z., Liu, Y., Han, N., Chen, X., Yu, W., Zhang, W., et al. (2010). Profiles of Oxidative Stress-Related microRNA and mRNA Expression in Auditory Cells. Brain Res. 1346, 14-25. doi:10.1016/j.brainres.2010.05.059

Wei, N., Xiao, L., Xue, R., Zhang, D., Zhou, J., au, H., et al. (2016). MicroRNA-9 Mediates the Cell Apoptosis by Targeting Bcl2l11 in Ischemic Stroke. Mol. Neurobiol. 53, 6809-6817. doi:10.1007/s12035-015-9605-4

Wu, Y., Xu, D., Zhu, X., Yang, G., and Ren, M. (2017). MiR-106a Associated with Diabetic Peripheral Neuropathy through the Regulation of 12/15-LOXMeidiated Oxidative/Nitrative Stress. Curr. Neurovasc Res. 14, 117-124. doi:10.2174/1567202614666170404115912

Xue, Y., Li, M., Liu, D., Zhu, Q., and Chen, H. (2018). Expression of miR-9 in the Serum of Patients with Acute Ischemic Stroke and its Effect on Neuronal Damage. Int. J. Clin. Exp. Pathol. 11, 5885-5892.

Yan, H. Y., Bu, S. Z., Zhou, W. B., and Mai, Y. F. (2018). TUG1 Promotes Diabetic Atherosclerosis by Regulating Proliferation of Endothelial Cells via Wnt 
Pathway. Eur. Rev. Med. Pharmacol. Sci. 22, 6922-6929. doi:10.26355/ eurrev_201810_16162

Yang, D., Yu, J., Liu, H.-B., Yan, X.-Q., Hu, J., Yu, Y., et al. (2019). The Long Noncoding RNA TUG1-miR-9a-5p axis Contributes to Ischemic Injuries by Promoting Cardiomyocyte Apoptosis via Targeting KLF5. Cel Death Dis 10, 908. doi:10.1038/s41419-019-2138-4

Zang, X. J., Li, L., Du, X., Yang, B., and Mei, C. L. (2019). LncRNA TUG1 Inhibits the Proliferation and Fibrosis of Mesangial Cells in Diabetic Nephropathy via Inhibiting the PI3K/AKT Pathway. Eur. Rev. Med. Pharmacol. Sci. 23, 7519-7525. doi:10.26355/eurrev_201909_18867

Zhang, L., Cheng, H., Yue, Y., Li, S., Zhang, D., and He, R. (2018). TUG1 Knockdown Ameliorates Atherosclerosis via Up-Regulating the Expression of miR-133a Target Gene FGF1. Cardiovasc. Pathol. 33, 6-15. doi:10.1016/j. carpath.2017.11.004

Zhu, D., Pan, C., Li, L., Bian, Z., Lv, Z., Shi, L., et al. (2013). MicroRNA-17/20a/106a Modulate Macrophage Inflammatory Responses through Targeting Signal-Regulatory Protein a. J. Allergy Clin. Immunol. 132, 426-436. doi:10.1016/j.jaci.2013.02.005
Conflict of Interest: The authors declare that the research was conducted in the absence of any commercial or financial relationships that could be construed as a potential conflict of interest.

Publisher's Note: All claims expressed in this article are solely those of the authors and do not necessarily represent those of their affiliated organizations, or those of the publisher, the editors and the reviewers. Any product that may be evaluated in this article, or claim that may be made by its manufacturer, is not guaranteed or endorsed by the publisher.

Copyright (c) 2022 Abdelaleem, Shaker, Mohamed, Ahmed, Elkhateeb, Abdelghaffar, Ahmed, Khalefa, Hemeda and Mahmoud. This is an open-access article distributed under the terms of the Creative Commons Attribution License (CC BY). The use, distribution or reproduction in other forums is permitted, provided the original author(s) and the copyright owner(s) are credited and that the original publication in this journal is cited, in accordance with accepted academic practice. No use, distribution or reproduction is permitted which does not comply with these terms. 